\title{
DISTILLATION METHODS AFFECT THE CHEMICAL COMPOSITION OF Varronia curassavica Jacq. ESSENTIAL OIL?
}

\author{
MÉTODOS DE DESTILAÇÃO AFETAM A COMPOSIÇÃO QUÍMICA DO ÓLEO \\ ESSENCIAL DE Varronia curassavica Jacq?
}

\author{
Daniela Aparecida de Castro NIZIO ${ }^{1}$; Arie Fitzgerald BLANK ${ }^{1}$; Taís Santos SAMPAIO²; \\ Fabiany de Andrade BRITO ${ }^{1}$; Thiago Matos ANDRADE ${ }^{1}$; \\ Maria de Fátima ARRIGONI-BLANK ${ }^{1}$; Alexandre Nizio MARIA ${ }^{3}$ \\ 1. Departamento de Engenharia Agronômica, Universidade Federal de Sergipe, São Cristóvão, SE, Brasil, \\ danielanizio82@gmail.com; 2. Departamento de Química, Universidade Federal de Sergipe, São Cristóvão, SE, Brasil; \\ 3. Laboratório de Reprodução Animal, Embrapa Tabuleiros Costeiros, Aracaju, SE, Brasil.
}

\begin{abstract}
The objective of this work was to evaluate the chemical composition of essential oil from Varronia curassavica Jacq. obtained by microwave (MI) and hydrodistillation (HD) extraction methods. The MI method tested three powers $(500,600$, and $700 \mathrm{~W})$, three distillation times $(20,30$, and $40 \mathrm{~min}$.$) , and three water volumes (0,25$, and $50 \mathrm{~mL}$ per sample). The HD method tested three distillation times (100, 120, and $140 \mathrm{~min}$.) and three water volumes (1.0, 1.5, and $2.0 \mathrm{~L}$ per 3-liter flask). The essential oils were analyzed by GC/MS-FID. The optimal condition for the essential oil extraction by the MI method was $700 \mathrm{~W}$ for $40 \mathrm{~min}$. (3.28\%), regardless of the volume of water. In its turn, the best condition for essential oil extraction by the HD method was $120 \mathrm{~min}$. with $1.0 \mathrm{~L}$ of water per flask $(3.34 \%)$. The most abundant compounds for MI (700 W for $40 \mathrm{~min}$. without water) were shyobunol $(26.53 \%)$ and bicyclogermacrene (4.96\%); and the most abundant compounds for HD (120 min. with $1.0 \mathrm{~L}$ of water/flask) were shyobunol (24.00\%) and germacrene D-4-ol (10.23\%). Methyl farnesoate $(2 E, 6 E)$ and farnesyl acetate $(2 Z, 6 E)$ were not detected in the essential oil extracted by HD; however, they were identified by the MI method. By increasing the distillation time and/or volume of water in HD, a reduction was observed for the content of the chemical compounds $\beta$-elemene (from 1.23 to $0.97 \%$ ), $E$ caryophyllene (from 5.49 to $4.35 \%$ ), $\alpha$-humulene (from 1.80 to $1.43 \%$ ), alloaromadendrene (from 1.78 to $1.44 \%$ ), bicyclogermacrene (from 5.63 to $4.55 \%$ ), and germacrene D-4-ol (from 11.40 to $9.86 \%$ ). Power, extraction time, and their interactions influenced the content of essential oil obtained by microwave extraction (MI). Within each power, the highest essential oil content was extracted at the longest distillation time (40 min.), except for $600 \mathrm{~W}$, where no significant difference was detected between 30 and $40 \mathrm{~min}$. The optimal essential oil contents for both extraction methods were statically similar by the t-test for dependent samples. However, the MI method presents advantages, such as shorter distillation time and less energy and water consumption.
\end{abstract}

KEYWORDS: Microwave distillation/ Volatile oil/ Chemical compounds

\section{INTRODUCTION}

The medicinal and aromatic plant Varronia curassavica Jacq. (syn. Cordia verbenacea DC.), known as "erva-baleeira" in Brazil, belongs to the family Cordiaceae (GASPARINO; BARROS, 2009) and is largely distributed in Brazil. Several properties of the species, such as anti-inflammatory, antiucer, antiallergic, antitumor, and antioxidant are mainly attributed to the compounds $\alpha$-humulene and $E$-caryophyllene, present in its essential oil (FERNANDES et al., 2007; PASSOS et al., 2007; MEDEIROS et al., 2007; ROGÉRIO et al., 2009; OLIVEIRA et al., 2011; MICHIELIN et al., 2011; PARISSOTO et al., 2012; PIMENTEL et al., 2012). Other biological activities, such as antibacterial (MECCIA et al., 2009; MATIAS et al., 2010; PINHO et al., 2012; RODRIGUES et al., 2012) and antifungal activities (SILVA et al., 2012; HOYOS et al., 2012; SILVA et al., 2014) were reported in studies involving the essential oil of $V$. curassavica. In the pharmaceutical industry, the essential oil of $V$. curassavica is used to make a phytotherapeutic medicine for inflammation treatment.

Essential oils consist of a complex mixture of several chemical compounds with wide variation in their composition, and most of the compounds are terpenes. The chemical composition of essential oils is influenced by plants genetics, environmental factors (irrigation, harvest time, season, and others), genotype $\mathrm{x}$ environment interaction, and processing parameters (e.g., temperature, drying time of the plant material, and essential oil extraction method) (VERMA et al., 2010; GONZÁLEZ-RIVERA et al., 2016). The extraction method is one of the primary factors to determine the quality of an essential oil. The improper extraction process can result in changes in the chemical composition of the essential oil, leading to the loss of its bioactivity and natural 
characteristics, such as color, flavor, and viscosity (TONGNUANCHAN; BENJAKUL, 2014).

Among the laboratory methods used to extract essential oils from plants, hydrodistillation (HD) is the most frequently applied. However, it presents disadvantages, such as long extraction time, high demand for energy and water, and overheating of plant material, causing the loss of bioactive compounds of the essential oil by thermal degradations (RANITHA et al., 2014; CHEN et al., 2016).

New techniques are currently available, such as microwave-assisted extraction (MI). This technique allows the plant material to reach its boiling point quickly, resulting in a shorter extraction time and with less energy expenditure (KHANAVI et al., 2013; LI et al., 2014). In this sense, several works have been carried out aiming to improve the conditions of microwave-assisted extraction for several medicinal and commercial species, such as Mentha spp. (COSTA et al., 2014; GAVAHIAN et al., 2015); Rosmarinus officinalis (KARAKAYA et al., 2014); Eucalyptus camaldulensis (LI et al., 2016); Cinnamomum cassia (CHEN et al., 2016), among others.

The objective of this work was to evaluate the content (\%) and chemical composition of essential oil from $V$. curassavica obtained by the hydrodistillation (HD) and microwave (MI) extraction methods.

\section{MATERIAL AND METHODS}

For essential oil extraction, leaves were harvested from plants of Varronia curassavica Jacq. (accession VCUR-201), maintained at the Active Germplasm Bank (AGB) of aromatic plants of the Federal University of Sergipe, located at the Research Farm "Campus Rural da UFS". Fresh leaves from the entire plants were collected, weighted, separated for essential oil extraction, maintained in a freezer at $-20^{\circ} \mathrm{C}$, until the beginning of the experiments.

The hydrodistillation was performed in a modified Clevenger apparatus using 3-liter flasks and samples of $100 \mathrm{~g}$ of fresh leaves. The experiment consisted of a completely randomized design, in a $3 \times 3$ factorial scheme, using three replications. Three distillation times $(100,120$, and 140 minutes of boiling) and three volumes of filtered water (using osmose reverse filter system) (1.0, 1.5, and $2.0 \mathrm{~L}$ per flask) were tested. The essential oils were dried over anhydrous sodium sulfate and stored in amber vials at $-20^{\circ} \mathrm{C}$, until chemical composition analysis.
The essential oil content was calculated using the mean dry weight of six samples of $100 \mathrm{~g}$ of fresh leaves at $100^{\circ} \mathrm{C}$ in a forced-air-circulation oven until constant weight, for 48 hours. The essential oil content was calculated according to the following equation:

Content $(\%, v / w)=($ volume of essential oil extracted from the sample/mean of dry weight of leaves) x 100 .

The microwave-accelerated reaction system (NEOS, Milestone, Italy) equipped with a circulating water-cooling system was utilized for the experiment. Samples of $50 \mathrm{~g}$ of fresh leaves were used. The experiment consisted of a completely randomized design, in a $3 \times 3 \times 3$ factorial scheme, with three replications. Three powers $(500,600$, and $700 \mathrm{~W})$, three extraction times $(20,30$, and 40 minutes), and three volumes of filtered water (using osmose reverse filter system) $(0,25$, and $50 \mathrm{~mL}$ per sample) were tested. The essential oils were dried using anhydrous sodium sulfate and stored in amber vials at $-20{ }^{\circ} \mathrm{C}$ until chemical composition analysis. The same procedure described for hydrodistillation was applied to calculate the essential oil content.

The analysis of the chemical composition of the essential oils was performed using a GCMS/FID (QP2010 Ultra, Shimadzu Corporation, Kyoto, Japan) equipped with an autosampler AOC20i (Shimadzu). Separations were accomplished using an Rtx®-5MS Restek fused silica capillary column (5\%-diphenyl-95\%-dimethyl polysiloxane) of $30 \mathrm{~m} \times 0.25 \mathrm{~mm}$ i.d., $0.25 \mathrm{~mm}$ film thickness, at a constant helium $(99.999 \%)$ flow rate of $1.2 \mathrm{~mL} \cdot \mathrm{min}^{-}$ 1. An injection volume of $0.5 \mu \mathrm{L}\left(5 \mathrm{mg} \cdot \mathrm{mL}^{-1}\right)$ was employed, with a split ratio of 1:10. The oven temperature was programmed from $50^{\circ} \mathrm{C}$ (isothermal for $1.5 \mathrm{~min}$ ), with an increase of 4 ${ }^{\circ} \mathrm{C} \cdot \mathrm{min}^{-1}$, to $200{ }^{\circ} \mathrm{C}$, then $10{ }^{\circ} \mathrm{C} \cdot \mathrm{min}^{-1}$ to $250{ }^{\circ} \mathrm{C}$, ending with a $5 \mathrm{~min}$ isothermal at $250{ }^{\circ} \mathrm{C}$.

The MS and FID data were simultaneously acquired by a Detector Splitting System; the split flow ratio was $4: 1$ (MS:FID). A $0.62 \mathrm{~m} \times 0.15 \mathrm{~mm}$ i.d. restrictor tube (capillary column) was used to connect the splitter to the MS detector; a $0.74 \mathrm{~m} \mathrm{x}$ $0.22 \mathrm{~mm}$ i.d. restrictor tube was used to connect the splitter to the FID detector. The MS data (total ion chromatogram, TIC) were obtained in the full scan mode $(\mathrm{m} / \mathrm{z}$ of $40-350)$ at a $0.3 \mathrm{scan} / \mathrm{s}$ scan rate, using the electron ionization (EI) with an electron energy of $70 \mathrm{eV}$. The injector temperature was 250 ${ }^{\circ} \mathrm{C}$, and the ion-source temperature was $250{ }^{\circ} \mathrm{C}$. The FID temperature was set to $250{ }^{\circ} \mathrm{C}$, and the gas supplies for the FID were hydrogen, air, and helium at flow rates of 30,300 , and $30 \mathrm{~mL} \cdot \mathrm{min}^{-1}$, respectively. Quantification of each constituent was 
estimated by FID peak-area normalization (\%). Compound concentrations were calculated from the GC peak areas and arranged in order of GC elution.

The identification of individual components of the essential oil was performed by computerized matching of the acquired mass spectra with those stored in the NIST21, NIST107, and WILEY8 mass spectral library of the GC-MS data system. A mixture of hydrocarbons $\left(\mathrm{C}_{9} \mathrm{H}_{20}-\mathrm{C}_{19} \mathrm{H}_{40}\right)$ was injected under these same conditions. Compounds were identified by comparing the spectra obtained with those of the equipment's data bank and by the Kovats index, calculated for each compound, as previously described (ADAMS, 2007). Retention indices were obtained using the equation proposed by Van den Dool and Kratz (1963).

Essential oil content and chemical composition were subject to analysis of variance (ANOVA), and the means were compared by the Scott-Knott test $(\mathrm{p}<0.05)$, using the Sisvar ${ }^{\circledR}$ software. The means of the content of the best hydrodistillation (HD) and microwave (MI) treatments were compared by the t-test for dependent samples $(\mathrm{p}<0.05)$, using the Statistica 7.0 software.

\section{RESULTS}

The optimal condition for essential oil extraction by the MI method was $700 \mathrm{~W}$ for $40 \mathrm{~min}$. $(3.28 \%)$, regardless of the volume of water. In its turn, the best condition for essential oil extraction by the HD method was $120 \mathrm{~min}$. with $1.0 \mathrm{~L}$ of water per flask (3.34\%). (Tables 1 and 2). The most abundant compounds for MI (700W for $40 \mathrm{~min}$. without water) were shyobunol (26.53\%) and bicyclogermacrene $(4.96 \%)$; and the most abundant compounds for HD (120 min. with $1.0 \mathrm{~L}$ of water/flask) were shyobunol $(24.00 \%)$ and germacrene D-4-ol (10.23\%) (Tables 1 and 2).

Most of the compounds presented significant distillation time $\mathrm{x}$ water volume interaction for HD. The increase in the distillation time and/or volume of water reduced the content of the chemical compounds $\beta$-elemene (from 1.23 to $0.97 \%$ ), $E$-caryophyllene (from 5.49 to $4.35 \%$ ), $\alpha$ humulene (from 1.80 to $1.43 \%$ ), alloaromadendrene (from 1.78 to $1.44 \%$ ), bicyclogermacrene (from 5.63 to $4.55 \%$ ), and germacrene D-4-ol (from 11.40 to $9.86 \%$ ) (Table 1). The content of germacrene D-4-ol reduced when distillation time was increased. However, the largest volume of water per flask resulted in higher contents, reaching the maximum $(12.55 \%)$ with 100 minutes distillation time and 2.0
$\mathrm{L}$ of water per flask. The contents of the compounds spathulenol, ledol, epi- $\alpha$-murulol, and shyobunol increased with the increase in the volume of water and/or distillation time (Table 1).

Power, extraction time, and their interactions influenced the essential oil content obtained by microwave extraction (MI). Within each power, the highest essential oil content was obtained at the longest extraction time (40 min.), except for $600 \mathrm{~W}$, where no significant difference was recorded between 30 and 40 minutes. The maximum essential oil content (3.28\%) was observed using the power of $700 \mathrm{~W}$ for $40 \mathrm{~min}$., regardless of the volume of water/sample (Table 2).

Methyl farnesoate $(2 E, 6 E)$ and farnesyl acetate $(2 Z, 6 E)$ were not detected in the essential oil extracted by HD. However, they were detected by the MI extraction method.

The extraction time of $40 \mathrm{~min}$. used in the solvent-free MI distillation together with the highest power $(700 \mathrm{~W})$ resulted in an increased content of $\beta$ elemene $(0.99 \%), \quad$ E-caryophyllene $(3.97 \%), \alpha-$ humulene $(1.44 \%)$, alloaromadendrene $(1.65 \%)$, bicyclogermacrene $(4.96 \%)$, ledol $(4.00 \%)$, epi- $\alpha-$ murulol $(4.06 \%)$, and farnesyl acetate $(2 Z, 6 E)$ (3.59\%) (Table 2). Only the content of alloaromadendrene $(1.34 \%)$, ledol $(4.10 \%)$, and farnesyl acetate $(2 Z, 6 E)(3.60 \%)$ increased with the addition of $50 \mathrm{~mL}$ of water per sample to the MI extraction system (Table 2).

The extraction time of $40 \mathrm{~min}$. in the solvent-free MI distillation together with the lowest power $(500 \mathrm{~W})$ resulted in an increased content of germacrene D-4-ol (4.24\%), caryophyllene oxide (2.24\%), cubenol (2.17\%), shyobunol $(27.35 \%)$, and methyl farnesoate $(2 E, 6 E)(3.51 \%)$ (Table 2). Only the content of germacrene D-4-ol (5.51\%) and methyl farnesoate $(2 E, 6 E)(3.11 \%)$ increased with the addition of $50 \mathrm{~mL}$ of water per sample to the MI extraction system (Table 2 ).

The means of essential oil contents obtained in the best HD $(120 \mathrm{~min}$. with $1.0 \mathrm{~L}$ of water per flask) and MI (700W for $40 \mathrm{~min}$ ) treatments were statistically similar $(\mathrm{p}<0.05)$ when compared by the $\mathrm{t}$-test for dependent samples. 
Table 1. Content and chemical composition of the essential oil from Varronia curassavica extracted by hydrodistillation (HD), according to the extraction time and water volume.

\begin{tabular}{|c|c|c|c|c|c|c|}
\hline \multirow{3}{*}{ Compounds } & \multirow{3}{*}{ RRI-o } & \multirow{3}{*}{ RRI-I } & \multirow{3}{*}{$\begin{array}{l}\text { Time } \\
\text { (min.) }\end{array}$} & \multicolumn{3}{|c|}{ Water (L/flask) } \\
\hline & & & & 1.0 & 1.5 & 2.0 \\
\hline & & & & \multicolumn{3}{|c|}{ Content (\%) of chemical constituents } \\
\hline \multirow{3}{*}{$\beta$-elemene } & \multirow{3}{*}{1389} & \multirow{3}{*}{1389} & 100 & $1.23 \mathrm{aA}$ & $1.15 \mathrm{aA}$ & $1.18 \mathrm{aA}$ \\
\hline & & & 120 & $1.21 \mathrm{aA}$ & $1.11 \mathrm{aB}$ & $1.10 \mathrm{bB}$ \\
\hline & & & 140 & $1.14 \mathrm{bA}$ & $1.09 \mathrm{aA}$ & $0.97 \mathrm{cB}$ \\
\hline \multirow{3}{*}{$E$-caryophyllene } & \multirow{3}{*}{1422} & \multirow{3}{*}{1417} & 100 & $5.49 \mathrm{aA}$ & $5.14 \mathrm{aB}$ & $5.20 \mathrm{aB}$ \\
\hline & & & 120 & $5.44 \mathrm{aA}$ & $4.98 \mathrm{aB}$ & $4.87 \mathrm{bB}$ \\
\hline & & & 140 & $4.98 \mathrm{aA}$ & $4.92 \mathrm{aA}$ & $4.35 \mathrm{cB}$ \\
\hline \multirow{3}{*}{$\alpha$-humulene } & \multirow{3}{*}{1457} & \multirow{3}{*}{1452} & 100 & $1.80 \mathrm{aA}$ & $1.71 \mathrm{aB}$ & $1.7 \mathrm{aB}$ \\
\hline & & & 120 & $1.76 \mathrm{aA}$ & $1.64 \mathrm{aB}$ & $1.61 \mathrm{bB}$ \\
\hline & & & 140 & $1.63 \mathrm{bA}$ & $1.61 \mathrm{bA}$ & $1.43 \mathrm{cB}$ \\
\hline \multirow{3}{*}{ alloaromadendrene } & \multirow{3}{*}{1465} & \multirow{3}{*}{1458} & 100 & $1.78 \mathrm{aA}$ & $1.66 \mathrm{aB}$ & $1.67 \mathrm{aB}$ \\
\hline & & & 120 & $1.74 \mathrm{aA}$ & $1.63 \mathrm{aB}$ & $1.56 \mathrm{bB}$ \\
\hline & & & 140 & $1.69 \mathrm{aA}$ & $1.69 \mathrm{aA}$ & $1.44 \mathrm{cB}$ \\
\hline \multirow{3}{*}{ bicyclogermacrene } & \multirow{3}{*}{1499} & \multirow{3}{*}{1500} & 100 & $5.63 \mathrm{aA}$ & $5.27 \mathrm{aB}$ & $5.44 \mathrm{aB}$ \\
\hline & & & 120 & $5.31 \mathrm{bA}$ & $5.20 \mathrm{aA}$ & $4.91 \mathrm{bB}$ \\
\hline & & & 140 & $5.01 \mathrm{cA}$ & $5.00 \mathrm{aA}$ & $4.55 \mathrm{cB}$ \\
\hline \multirow{3}{*}{ germacrene D-4-ol } & & & 100 & $11.40 \mathrm{aB}$ & $11.70 \mathrm{aB}$ & $12.55 \mathrm{aA}$ \\
\hline & 1580 & 1574 & 120 & $10.23 \mathrm{bC}$ & $11.09 \mathrm{bB}$ & $11.84 \mathrm{bA}$ \\
\hline & & & 140 & $9.86 \mathrm{bB}$ & $9.90 \mathrm{cB}$ & $10.36 \mathrm{cA}$ \\
\hline & & & 100 & $1.16 \mathrm{aB}$ & $1.20 \mathrm{aB}$ & $1.32 \mathrm{aA}$ \\
\hline sphatulenol & 1583 & 1577 & 120 & $1.10 \mathrm{aA}$ & $1.19 \mathrm{aA}$ & $1.21 \mathrm{bA}$ \\
\hline & & & 140 & $1.11 \mathrm{aA}$ & $1.14 \mathrm{aA}$ & $1.13 \mathrm{bA}$ \\
\hline & & & 100 & $1.34 \mathrm{aA}$ & $1.34 \mathrm{aA}$ & $0.97 \mathrm{bB}$ \\
\hline caryophyllene oxide & 1592 & 1582 & 120 & $1.36 \mathrm{aA}$ & $1.34 \mathrm{aA}$ & $1.34 \mathrm{aA}$ \\
\hline & & & 140 & $1.32 \mathrm{aA}$ & $1.35 \mathrm{aA}$ & $1.34 \mathrm{aA}$ \\
\hline & & & 100 & $3.71 \mathrm{aB}$ & $3.65 \mathrm{bB}$ & $4.50 \mathrm{aA}$ \\
\hline ledol & 1612 & 1602 & 120 & $3.69 \mathrm{aB}$ & $3.88 \mathrm{aA}$ & $3.93 \mathrm{bA}$ \\
\hline & & & 140 & $3.67 \mathrm{aB}$ & $3.67 \mathrm{bB}$ & $3.88 \mathrm{bA}$ \\
\hline & & & 100 & $2.76 \mathrm{bB}$ & $2.90 \mathrm{bB}$ & $3.90 \mathrm{aA}$ \\
\hline epi- $\alpha$-murulol & 1646 & 1640 & 120 & $3.00 \mathrm{bB}$ & $4.10 \mathrm{aA}$ & $3.97 \mathrm{aA}$ \\
\hline & & & 140 & $3.63 \mathrm{aB}$ & $3.94 \mathrm{aA}$ & $3.92 \mathrm{aA}$ \\
\hline & & & 100 & $1.82 \mathrm{aA}$ & $1.86 \mathrm{aA}$ & $1.75 \mathrm{bA}$ \\
\hline cubenol & 1650 & 1645 & 120 & $1.85 \mathrm{aA}$ & $2.00 \mathrm{aA}$ & $1.89 \mathrm{bA}$ \\
\hline & & & 140 & $1.98 \mathrm{aA}$ & $2.06 \mathrm{aA}$ & $2.22 \mathrm{aA}$ \\
\hline & & & 100 & $4.56 \mathrm{aA}$ & $4.68 \mathrm{aA}$ & $4.83 \mathrm{bA}$ \\
\hline$\alpha$-cadinol & 1660 & 1652 & 120 & $4.91 \mathrm{aA}$ & $4.93 \mathrm{aA}$ & $5.33 \mathrm{aA}$ \\
\hline & & & 140 & $4.94 \mathrm{aA}$ & $5.41 \mathrm{aA}$ & $5.63 \mathrm{aA}$ \\
\hline & & & 100 & $24.24 \mathrm{bA}$ & $23.90 \mathrm{bA}$ & $22.95 \mathrm{cB}$ \\
\hline shyobunol & 1705 & 1709 & 120 & $24.00 \mathrm{bA}$ & $24.14 \mathrm{bA}$ & $24.24 \mathrm{bA}$ \\
\hline & & & 140 & $24.93 \mathrm{aB}$ & $24.81 \mathrm{aB}$ & $25.58 \mathrm{aA}$ \\
\hline & & & 100 & $2.95 \mathrm{aA}$ & $3.10 \mathrm{aA}$ & $1.67 \mathrm{cB}$ \\
\hline methyl farnesoate $(2 E, 6 E)$ & 1776 & 1783 & 120 & $2.25 \mathrm{bB}$ & $2.91 \mathrm{aA}$ & $2.88 \mathrm{aA}$ \\
\hline & & & 140 & $2.86 \mathrm{aA}$ & $2.77 \mathrm{aA}$ & $2.24 \mathrm{bB}$ \\
\hline & & & 100 & $2.93 \mathrm{bA}$ & $2.93 \mathrm{bA}$ & $2.43 \mathrm{cB}$ \\
\hline essential oil content (\%) & --- & --- & 120 & $3.34 \mathrm{aA}$ & $3.03 \mathrm{bB}$ & $2.93 \mathrm{bB}$ \\
\hline & & & 140 & $3.54 \mathrm{aA}$ & $3.44 \mathrm{aA}$ & $3.23 \mathrm{aA}$ \\
\hline
\end{tabular}

RRI-o: Relative Retention Index - observed; RRI-l: Relative Retention Index - literature. Means followed by the same lowercase letter in the column and uppercase letter in the row do not differ by the Scott Knott test $(\mathrm{P}<0.05)$. 
Table 2. Content and chemical composition of the essential oil from Varronia curassavica obtained by solvent-free microwave extraction (MI), according to the tested power, extraction time, and water volume.

\begin{tabular}{|c|c|c|c|c|c|c|c|c|c|c|c|c|}
\hline \multirow{4}{*}{ Compounds } & \multirow{4}{*}{ RRI-o } & \multirow{4}{*}{ RRI-l } & \multirow{4}{*}{ Power $(W)$} & \multicolumn{9}{|c|}{ Water (mL/sample) } \\
\hline & & & & \multirow{2}{*}{\multicolumn{9}{|c|}{$\frac{0}{\text { Time (min.) }}$}} \\
\hline & & & & & & & & & & & & \\
\hline & & & & 20 & 30 & 40 & 20 & 30 & 40 & 20 & 30 & 40 \\
\hline & & & & \multicolumn{9}{|c|}{ Content $(\%)$ of chemical compounds } \\
\hline \multirow{3}{*}{$\beta$-elemene } & \multirow{3}{*}{1389} & \multirow{3}{*}{1389} & 500 & $1.05 \mathrm{bA} \beta$ & $1.01 \mathrm{bA} \alpha$ & $0.96 \mathrm{aB} \alpha$ & $1.00 \mathrm{bA} \beta$ & $0.89 \mathrm{cB} \beta$ & $0.87 \mathrm{cB} \beta$ & $1.20 \mathrm{aA} \alpha$ & $0.83 \mathrm{cB} \beta$ & $0.87 \mathrm{aB} \beta$ \\
\hline & & & 600 & $1.21 \mathrm{aA} \alpha$ & $1.12 \mathrm{aB} \alpha$ & $0.87 \mathrm{bC} \beta$ & $1.19 \mathrm{aA} \alpha$ & $1.13 \mathrm{aA} \alpha$ & $1.03 \mathrm{aB} \alpha$ & $1.08 \mathrm{bA} \beta$ & $1.02 \mathrm{aA} \beta$ & $0.93 \mathrm{aB} \beta$ \\
\hline & & & 700 & $1.25 \mathrm{aA} \alpha$ & $1.02 \mathrm{bB} \alpha$ & $0.99 \mathrm{aB} \alpha$ & $1.03 \mathrm{bA} \beta$ & $1.03 \mathrm{bA} \alpha$ & $0.94 \mathrm{bB} \alpha$ & $1.00 \mathrm{cA} \beta$ & $0.93 \mathrm{bA} \beta$ & $0.93 \mathrm{aA} \alpha$ \\
\hline \multirow{3}{*}{$E$-caryophyllene } & \multirow{3}{*}{1422} & \multirow{3}{*}{1417} & 500 & $4.40 \mathrm{aA} \gamma$ & $3.55 \mathrm{cB} \alpha$ & $3.43 \mathrm{bB} \alpha$ & $3.78 \mathrm{bA} \beta$ & $2.87 \mathrm{bB} \beta$ & $2.92 \mathrm{bB} \beta$ & $5.24 \mathrm{aA} \alpha$ & $2.60 \mathrm{bC} \gamma$ & $3.08 \mathrm{aB} \beta$ \\
\hline & & & 600 & $4.45 \mathrm{aA} \alpha$ & $4.19 \mathrm{aB} \alpha$ & $2.99 \mathrm{cC} \beta$ & $4.25 \mathrm{aA} \alpha$ & $3.82 \mathrm{aB} \beta$ & $3.59 \mathrm{aC} \alpha$ & $3.82 \mathrm{bA} \beta$ & $3.51 \mathrm{aB} \gamma$ & $3.19 \mathrm{aC} \beta$ \\
\hline & & & 700 & $4.59 \mathrm{aA} \alpha$ & $3.91 \mathrm{bB} \alpha$ & $3.97 \mathrm{aB} \alpha$ & $3.96 \mathrm{bA} \beta$ & $3.68 \mathrm{aB} \beta$ & $3.46 \mathrm{aB} \beta$ & $3.67 \mathrm{bA} \gamma$ & $3.35 \mathrm{aB} \gamma$ & $3.12 \mathrm{aC} \gamma$ \\
\hline \multirow{3}{*}{$\alpha$-humulene } & \multirow{3}{*}{1457} & \multirow{3}{*}{1452} & 500 & $1.49 \mathrm{bA} \beta$ & $1.30 \mathrm{cB} \alpha$ & $1.29 \mathrm{bB} \alpha$ & $1.36 \mathrm{cC} \gamma$ & $1.14 \mathrm{bB} \beta$ & $1.15 \mathrm{bB} \beta$ & $1.75 \mathrm{aA} \alpha$ & $1.06 \mathrm{bC \gamma}$ & $1.19 \mathrm{aB} \beta$ \\
\hline & & & 600 & $1.58 \mathrm{bA \alpha}$ & $1.51 \mathrm{aB} \alpha$ & $1.19 \mathrm{cC} \beta$ & $1.56 \mathrm{aA} \alpha$ & $1.45 \mathrm{aB} \alpha$ & $1.36 \mathrm{aC} \alpha$ & $1.42 \mathrm{bA} \beta$ & $1.37 \mathrm{aA} \beta$ & $1.25 \mathrm{aB} \beta$ \\
\hline & & & 700 & $1.68 \mathrm{aA} \alpha$ & $1.39 \mathrm{bB} \alpha$ & $1.44 \mathrm{aB} \alpha$ & $1.44 \mathrm{bA} \beta$ & $1.40 \mathrm{aA} \alpha$ & $1.30 \mathrm{aB} \beta$ & $1.35 \mathrm{cA} \gamma$ & $1.31 \mathrm{aA} \beta$ & $1.21 \mathrm{aB} \gamma$ \\
\hline \multirow{3}{*}{ alloaromadendrene } & \multirow{3}{*}{1465} & \multirow{3}{*}{1458} & 500 & $1.65 \mathrm{cA} \alpha$ & $1.43 \mathrm{bB} \alpha$ & $1.45 \mathrm{bB} \alpha$ & $1.44 \mathrm{cA} \beta$ & $1.25 \mathrm{bB} \beta$ & $1.25 \mathrm{bB} \beta$ & $1.71 \mathrm{aA} \alpha$ & $1.11 \mathrm{cC} \gamma$ & $1.26 \mathrm{bB} \beta$ \\
\hline & & & 600 & $1.75 \mathrm{bA} \alpha$ & $1.65 \mathrm{aB} \alpha$ & $1.35 \mathrm{cC} \beta$ & $1.71 \mathrm{aA} \alpha$ & $1.54 \mathrm{aB} \beta$ & $1.52 \mathrm{aB} \alpha$ & $1.52 \mathrm{bA} \beta$ & $1.51 \mathrm{aA} \beta$ & $1.40 \mathrm{aB} \beta$ \\
\hline & & & 700 & $1.92 \mathrm{aA} \alpha$ & $1.59 \mathrm{aB} \alpha$ & $1.65 \mathrm{aB} \alpha$ & $1.62 \mathrm{bA} \beta$ & $1.57 \mathrm{aA} \alpha$ & $1.45 \mathrm{aB} \beta$ & $1.45 \mathrm{cA} \gamma$ & $1.44 \mathrm{bA} \beta$ & $1.34 \mathrm{aB} \gamma$ \\
\hline \multirow{3}{*}{ bicyclogermacrene } & \multirow{3}{*}{1499} & \multirow{3}{*}{1500} & 500 & $5.08 \mathrm{cA} \beta$ & $4.52 \mathrm{bB} \alpha$ & $4.59 \mathrm{bB} \alpha$ & $4.70 \mathrm{cA} \gamma$ & $4.25 \mathrm{bB} \beta$ & $4.20 \mathrm{cB} \beta$ & $5.31 \mathrm{aA} \alpha$ & $4.04 \mathrm{cC} \gamma$ & $4.22 \mathrm{bB} \beta$ \\
\hline & & & 600 & $5.41 \mathrm{bA \alpha}$ & $5.07 \mathrm{aB} \alpha$ & $4.41 \mathrm{cC} \beta$ & $5.31 \mathrm{aA} \alpha$ & $4.88 \mathrm{aB} \beta$ & $4.79 \mathrm{aB} \alpha$ & $4.88 \mathrm{bA} \beta$ & $4.71 \mathrm{aB} \gamma$ & $4.43 \mathrm{aC} \beta$ \\
\hline & & & 700 & $5.67 \mathrm{aA} \alpha$ & $4.94 \mathrm{aB} \alpha$ & $4.96 \mathrm{aB} \alpha$ & $5.07 \mathrm{bA} \beta$ & $4.82 \mathrm{aB} \alpha$ & $4.50 \mathrm{bC} \beta$ & $4.78 \mathrm{bA} \gamma$ & $4.50 \mathrm{bB} \beta$ & $4.21 \mathrm{bC} \gamma$ \\
\hline \multirow{3}{*}{ germacrene D-4-ol } & \multirow{3}{*}{1580} & \multirow{3}{*}{1574} & 500 & $4.09 \mathrm{aB} \gamma$ & $4.29 \mathrm{aA} \gamma$ & $4.24 \mathrm{aA} \gamma$ & $4.31 \mathrm{bC} \beta$ & $4.48 \mathrm{aB} \beta$ & $4.77 \mathrm{aA} \beta$ & $11.86 \mathrm{aA} \alpha$ & $5.09 \mathrm{aC} \alpha$ & $5.51 \mathrm{aB} \alpha$ \\
\hline & & & 600 & $4.03 \mathrm{aA} \gamma$ & $3.89 \mathrm{bB} \gamma$ & $4.02 \mathrm{bA} \gamma$ & $4.56 \mathrm{aA} \beta$ & $4.34 \mathrm{bB} \beta$ & $4.53 \mathrm{bA} \beta$ & $4.83 \mathrm{bA \alpha} \alpha$ & $4.75 \mathrm{bA} \alpha$ & $4.77 \mathrm{bA} \alpha$ \\
\hline & & & 700 & $3.93 \mathrm{aA} \gamma$ & $3.91 \mathrm{bA} \gamma$ & $3.60 \mathrm{cB} \gamma$ & $4.03 \mathrm{cB} \beta$ & $4.21 \mathrm{cA} \beta$ & $4.03 \mathrm{cB} \beta$ & $4.70 \mathrm{cA} \alpha$ & $4.62 \mathrm{cB} \alpha$ & $4.73 \mathrm{bA \alpha}$ \\
\hline \multirow{3}{*}{ caryophyllene oxide } & \multirow{3}{*}{1592} & & 500 & $2.45 \mathrm{aA} \alpha$ & $2.34 \mathrm{aB} \alpha$ & $2.24 \mathrm{aC} \alpha$ & $2.49 \mathrm{aA} \alpha$ & $2.14 \mathrm{bB} \beta$ & $2.20 \mathrm{aB} \alpha$ & $1.39 \mathrm{bC} \beta$ & $2.09 \mathrm{aB} \beta$ & $2.20 \mathrm{aA} \alpha$ \\
\hline & & 1582 & 600 & $2.46 \mathrm{aA} \alpha$ & $2.18 \mathrm{bC} \alpha$ & $2.28 \mathrm{aB} \alpha$ & $2.37 \mathrm{bA} \beta$ & $1.96 \mathrm{cC} \beta$ & $2.12 \mathrm{bB} \beta$ & $2.14 \mathrm{aA} \gamma$ & $2.12 \mathrm{aA} \alpha$ & $2.02 \mathrm{bB} \gamma$ \\
\hline & & & 700 & $2.28 \mathrm{bA} \alpha$ & $2.22 \mathrm{bA} \beta$ & $1.97 \mathrm{bB} \beta$ & $2.10 \mathrm{cB} \gamma$ & $2.30 \mathrm{aA} \alpha$ & $2.04 \mathrm{cB} \beta$ & $2.17 \mathrm{aA} \beta$ & $2.06 \mathrm{aA} \gamma$ & $2.17 \mathrm{aA} \alpha$ \\
\hline & & & 500 & $3.78 \mathrm{bA} \beta$ & $3.38 \mathrm{bB} \beta$ & $3.47 \mathrm{bB} \alpha$ & $3.71 \mathrm{cA} \beta$ & $3.79 \mathrm{bA} \alpha$ & $3.63 \mathrm{aA} \alpha$ & $4.01 \mathrm{bA} \alpha$ & $3.72 \mathrm{bB} \alpha$ & $3.62 \mathrm{bB} \alpha$ \\
\hline ledol & 1612 & 1602 & 600 & $3.62 \mathrm{bB} \beta$ & $4.03 \mathrm{aA} \beta$ & $3.98 \mathrm{aA} \alpha$ & $4.01 \mathrm{bA} \alpha$ & $3.93 \mathrm{bA} \beta$ & $3.86 \mathrm{aA} \alpha$ & $3.83 \mathrm{bB} \alpha$ & $4.30 \mathrm{aA} \alpha$ & $4.11 \mathrm{aA} \alpha$ \\
\hline & & & 700 & $4.22 \mathrm{aA} \alpha$ & $3.95 \mathrm{aB} \beta$ & $4.00 \mathrm{aB} \alpha$ & $4.26 \mathrm{aA} \alpha$ & $4.30 \mathrm{aA} \alpha$ & $3.87 \mathrm{aB} \alpha$ & $4.33 \mathrm{aA} \alpha$ & $4.10 \mathrm{aA} \beta$ & $4.10 \mathrm{aA} \alpha$ \\
\hline & & & 500 & $2.79 \mathrm{bB} \alpha$ & $2.88 \mathrm{bB} \gamma$ & $3.50 \mathrm{bA} \beta$ & $2.86 \mathrm{bC} \alpha$ & $4.16 \mathrm{aA} \alpha$ & $3.82 \mathrm{aB} \alpha$ & $2.98 \mathrm{bB} \alpha$ & $3.89 \mathrm{aA} \beta$ & $3.80 \mathrm{aA} \alpha$ \\
\hline epi- $\alpha$-murulol & 1646 & 1640 & 600 & $3.41 \mathrm{aB} \alpha$ & $3.67 \mathrm{aA} \alpha$ & $3.89 \mathrm{aA} \alpha$ & $3.58 \mathrm{aA} \alpha$ & $3.73 \mathrm{bA \alpha}$ & $3.68 \mathrm{aA} \alpha$ & $3.71 \mathrm{aA} \alpha$ & $3.69 \mathrm{aA} \alpha$ & $3.75 \mathrm{aA} \alpha$ \\
\hline & & & 700 & $3.60 \mathrm{aB} \alpha$ & $3.55 \mathrm{aB} \alpha$ & $4.06 \mathrm{aA} \alpha$ & $3.64 \mathrm{aA} \alpha$ & $3.79 \mathrm{bA} \alpha$ & $3.57 \mathrm{aA} \beta$ & $3.67 \mathrm{aA} \alpha$ & $3.75 \mathrm{aA} \alpha$ & $3.87 \mathrm{aA} \beta$ \\
\hline
\end{tabular}


Continuation of table 2:

\begin{tabular}{|c|c|c|c|c|c|c|c|c|c|c|c|c|}
\hline \multirow{3}{*}{ cubenol } & \multirow{3}{*}{1650} & \multirow{3}{*}{1645} & 500 & $1.63 \mathrm{aB} \alpha$ & $1.99 \mathrm{aA} \alpha$ & $2.17 \mathrm{aA} \alpha$ & $1.87 \mathrm{aA} \alpha$ & $1.77 \mathrm{aA} \alpha$ & $2.10 \mathrm{aA} \alpha$ & $1.93 \mathrm{aA} \alpha$ & $1.96 \mathrm{aA} \alpha$ & $1.57 \mathrm{bB} \beta$ \\
\hline & & & 600 & $1.90 \mathrm{aA} \alpha$ & $1.68 \mathrm{bA} \alpha$ & $2.02 \mathrm{aA} \alpha$ & $1.52 \mathrm{bB} \beta$ & $1.78 \mathrm{aA} \alpha$ & $1.89 \mathrm{aA} \alpha$ & $1.64 \mathrm{aA} \beta$ & $1.74 \mathrm{aA} \alpha$ & $1.86 \mathrm{aA} \alpha$ \\
\hline & & & 700 & $1.67 \mathrm{aA} \alpha$ & $2.07 \mathrm{aA} \alpha$ & $1.69 \mathrm{bB} \beta$ & $1.53 \mathrm{bB} \alpha$ & $1.70 \mathrm{aB} \beta$ & 2.09aA $\alpha$ & $1.78 \mathrm{aA} \alpha$ & $1.71 \mathrm{aA} \beta$ & $1.90 \mathrm{aA} \alpha$ \\
\hline \multirow{3}{*}{$\alpha$-cadinol } & \multirow{3}{*}{1660} & \multirow{3}{*}{1652} & 500 & $4.54 \mathrm{aA} \alpha$ & $4.51 \mathrm{aA} \alpha$ & $4.54 \mathrm{aA} \alpha$ & $4.17 \mathrm{aA} \alpha$ & $4.55 \mathrm{aA} \alpha$ & $4.26 \mathrm{aA} \alpha$ & $4.67 \mathrm{aA} \alpha$ & $4.62 \mathrm{aA} \alpha$ & $4.79 \mathrm{aA} \alpha$ \\
\hline & & & 600 & $3.84 \mathrm{aB} \beta$ & $4.49 \mathrm{aA} \alpha$ & $4.93 \mathrm{aA} \alpha$ & $4.55 \mathrm{aA} \alpha$ & $4.44 \mathrm{aA} \alpha$ & $4.31 \mathrm{aA} \beta$ & $4.01 \mathrm{aB} \beta$ & $4.62 \mathrm{aA} \alpha$ & $4.82 \mathrm{aA} \alpha$ \\
\hline & & & 700 & $4.50 \mathrm{aA} \alpha$ & $4.59 \mathrm{aA} \alpha$ & $4.28 \mathrm{aA} \alpha$ & $4.79 \mathrm{aA} \alpha$ & $4.24 \mathrm{aA} \alpha$ & $4.48 \mathrm{aA} \alpha$ & $4.19 \mathrm{aA} \alpha$ & $4.49 \mathrm{aA} \alpha$ & $4.60 \mathrm{aA} \alpha$ \\
\hline \multirow{3}{*}{ shyobunol } & \multirow{3}{*}{1705} & \multirow{3}{*}{1709} & 500 & $26.47 \mathrm{aB} \beta$ & $26.92 \mathrm{aA} \beta$ & $27.35 \mathrm{aA} \alpha$ & $27.30 \mathrm{aB} \alpha$ & $28.43 \mathrm{aA} \alpha$ & $27.84 \mathrm{aB} \alpha$ & $23.53 \mathrm{bC} \gamma$ & $29.07 \mathrm{aA} \alpha$ & $27.45 \mathrm{aB} \alpha$ \\
\hline & & & 600 & $26.11 \mathrm{aB} \beta$ & $25.88 \mathrm{bB} \beta$ & $27.22 \mathrm{aA} \alpha$ & $25.50 \mathrm{bB} \beta$ & $26.68 \mathrm{cA} \alpha$ & $26.91 \mathrm{bA} \alpha$ & $26.98 \mathrm{aA} \alpha$ & $26.40 \mathrm{cB} \alpha$ & $27.45 \mathrm{aA} \alpha$ \\
\hline & & & 700 & $25.20 \mathrm{bB} \beta$ & $26.11 \mathrm{bA} \beta$ & $26.53 \mathrm{bA} \beta$ & $27.34 \mathrm{aA} \alpha$ & $27.49 \mathrm{bA} \alpha$ & $27.12 \mathrm{bA} \alpha$ & $27.03 \mathrm{aA} \alpha$ & $27.71 \mathrm{bA} \alpha$ & $27.46 \mathrm{aA} \alpha$ \\
\hline \multirow{3}{*}{$\begin{array}{l}\text { methyl farnesoate } \\
(2 E, 6 E)\end{array}$} & \multirow{3}{*}{1776} & \multirow{3}{*}{1783} & 500 & $3.94 \mathrm{aB} \alpha$ & $4.55 \mathrm{aA} \alpha$ & $3.51 \mathrm{aC} \alpha$ & $3.33 \mathrm{aB} \beta$ & $3.67 \mathrm{aA} \beta$ & $3.19 \mathrm{aB} \beta$ & $1.79 \mathrm{cC} \gamma$ & $3.77 \mathrm{aA} \beta$ & $3.11 \mathrm{aB} \beta$ \\
\hline & & & 600 & $3.63 \mathrm{aA} \alpha$ & $3.83 \mathrm{bA \alpha}$ & $2.85 \mathrm{bB} \alpha$ & $2.44 \mathrm{bB} \gamma$ & $3.28 \mathrm{bA} \beta$ & $2.51 \mathrm{bB} \beta$ & $2.85 \mathrm{aA} \beta$ & $1.70 \mathrm{bB} \gamma$ & $1.84 \mathrm{bB} \gamma$ \\
\hline & & & 700 & $1.63 \mathrm{bA} \beta$ & $1.54 \mathrm{cA \alpha}$ & $1.78 \mathrm{cA} \alpha$ & $1.38 \mathrm{cB} \beta$ & $1.80 \mathrm{cA \alpha}$ & $2.10 \mathrm{cA} \alpha$ & $2.14 \mathrm{bA} \alpha$ & $1.63 \mathrm{bB} \alpha$ & $1.77 \mathrm{bB} \alpha$ \\
\hline \multirow{3}{*}{ farnesyl acetate $(2 Z, 6 E)$} & \multirow{3}{*}{1824} & \multirow{3}{*}{1821} & 500 & $2.50 \mathrm{cA} \beta$ & $2.16 \mathrm{cB} \alpha$ & $2.81 \mathrm{bA} \alpha$ & $2.83 \mathrm{cA} \alpha$ & $2.47 \mathrm{bA} \alpha$ & $2.72 \mathrm{cA} \alpha$ & $0.00 \mathrm{cC} \gamma$ & $2.03 \mathrm{bB} \beta$ & $2.40 \mathrm{bA} \beta$ \\
\hline & & & 600 & $2.82 \mathrm{bB} \beta$ & $2.69 \mathrm{bB} \beta$ & $3.56 \mathrm{aA} \alpha$ & $3.19 \mathrm{bA} \alpha$ & $2.48 \mathrm{bB} \beta$ & $3.24 \mathrm{bA} \beta$ & $2.70 \mathrm{bB} \beta$ & $3.64 \mathrm{aA} \alpha$ & $3.69 \mathrm{aA} \alpha$ \\
\hline & & & 700 & $4.34 \mathrm{aAa}$ & $3.83 \mathrm{aB} \alpha$ & $3.59 \mathrm{aB} \alpha$ & $4.14 \mathrm{aAa}$ & $3.61 \mathrm{aB} \alpha$ & $3.65 \mathrm{aB} \alpha$ & $3.33 \mathrm{aB} \beta$ & $3.83 \mathrm{aAa}$ & $3.60 \mathrm{aA} \alpha$ \\
\hline \multirow{3}{*}{ essential oil content $(\%)$} & \multirow{3}{*}{--- } & \multirow{3}{*}{---} & 500 & $1.65 \mathrm{bC} \alpha$ & $2.05 \mathrm{bB} \alpha$ & $2.69 \mathrm{bA} \alpha$ & $1.45 \mathrm{cC} \alpha$ & $2.07 \mathrm{bB} \alpha$ & $2.71 \mathrm{bA} \alpha$ & $1.52 \mathrm{cC} \alpha$ & $2.09 \mathrm{cB} \alpha$ & $2.58 \mathrm{cA} \alpha$ \\
\hline & & & 600 & $2.20 \mathrm{aB} \alpha$ & $2.86 \mathrm{aA} \alpha$ & $2.48 \mathrm{bB} \beta$ & $2.21 \mathrm{bB} \alpha$ & $2.88 \mathrm{aA} \alpha$ & $2.90 \mathrm{bA} \alpha$ & $2.08 \mathrm{bC} \alpha$ & $2.50 \mathrm{bB} \alpha$ & $3.00 \mathrm{bA} \alpha$ \\
\hline & & & 700 & $2.42 \mathrm{aB} \alpha$ & $2.90 \mathrm{aB} \alpha$ & $3.28 \mathrm{aAd}$ & $2.71 \mathrm{aB} \alpha$ & $2.90 \mathrm{aB} \alpha$ & $3.36 \mathrm{aA} \alpha$ & $2.71 \mathrm{aB} \alpha$ & $2.95 \mathrm{aB} \alpha$ & $3.43 \mathrm{aAa}$ \\
\hline
\end{tabular}

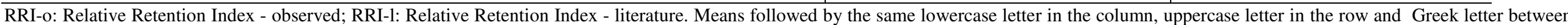
water volumes (for the same time and power) do not differ by the Scott Knott test $(\mathrm{P}<0.05)$. 


\section{DISCUSSION}

The use of smaller volumes of water in the extraction by HD provided the highest essential oil content. The addition of smaller volumes of water to the flasks allows more space for the movement of water vapor and essential oil during distillation, which favors higher essential oil condensing in the modified Clevenger apparatus. The compounds sesquiterpene hydrocarbons were observed in higher contents at a shorter distillation time and/or smaller volumes of water in the HD method. The increase in the extraction time led to a greater loss of sesquiterpene hydrocarbons compounds by volatilization and degradation due to the high temperature or hydrolysis (SOZMEN et al., 2011; LI et al., 2012; QI et al., 2014). Conversely, the oxygenated sesquiterpenes presented the highest contents when increasing the extraction time and/or volumes of water. Due to the lower volatility of these compounds, they are lost in a lesser amount when compared with the sesquiterpenes hydrocarbons during the extraction process.

The extraction time and power were the most important factors in the essential oil extraction of $V$. curassavica by MI. Similarly, Shah and Garg (2014) reported that the longest time (30 min.) and higher power $(640 \mathrm{~W})$ provided greater essential content in a study performed with ginger. According to Chen et al. (2011), the increase of power accelerates the transfer of mass and increases the essential oil content.

For the species Ocimum basilicum and Chenopodium ambrosioides, time and water factors were more significant than power for the essential oil content obtained by MI. Similarly to the present study for HD, Cardoso-Ugarte et al. (2013) reported that the longer the time of extraction and the lower the volumes of water, the greater were the essential oil contents. In their work, the authors used an adapted household microwave and larger volumes of water, which explains the resemblance of their results with that observed for the HD method in the current study.

A higher number of compounds was detected in the essential oil of $V$. curassavica extracted by the MI method when compared with that revealed by the HD method. The same was observed in Ferulago campestres (RIELA et al., 2011). The plant material heated by microwaves overheat inside the cell, which causes the expansion and rupture of the cell walls and consequently allows extracting the essential oil and less volatile compounds more efficiently (CHEN et al., 2011).

The significant interaction between the studied factors when using extraction by MI, for most compounds, showed that these factors cause quantitative variation in the chemical composition of the essential oil of $V$. curassavica. Similar germacrene D-4-ol content was observed between the essential oils extracted by HD and MI, where higher contents were reached when applying the shortest time and greater volumes of water. $E$ caryophyllene and $\alpha$-humulene presented similar behavior regarding the time, where higher contents were observed at shorter extraction times when using both methods. In a study carried out with clove (Syzygium aromaticum L.), a higher content of $E$-caryophyllene $(24.8 \%)$ and $\alpha$-humulene $(3.1 \%)$ were obtained by the MI method when compared with the hydrodistillation method (5.1 and $0.6 \%$, respectively) (GONZÁLEZ-RIVERA et al., 2016), probably due to the shorter time and lower volume of water used in the microwave-assisted extraction.

This study revealed that both methods (HD and MI) were effective in extracting the essential oil of $V$. curassavica. The chemical composition and essential oil content of this species are influenced by the extraction method used. The optimal essential oil contents for the two extraction methods were similar. However, the new technique of microwaveassisted extraction (MI) has some advantages, such as shorter distillation time and lower energy and water consumption. The use of $700 \mathrm{~W}$ for $40 \mathrm{~min}$. (without water) for MI extraction and $120 \mathrm{~min}$. with $1.0 \mathrm{~L}$ of water per flask for HD can be recommended to obtain high essential oil content of V. curassavica.

\section{ACKNOWLEDGMENTS}

The authors thank CNPq, FAPITEC/SE, CAPES, FINEP, and RENORBIO for their financial support of this work.

RESUMO: O objetivo deste trabalho foi avaliar a composição química do óleo essencial de Varronia curassavica Jacq. obtido pelos métodos de extração micro-ondas (MI) e hidrodestilação (HD). Para MI, foram testadas três potências $(500,600$ e $700 \mathrm{~W})$, três tempos de destilação $(20,30$ e 40 min.) e três volumes de água $(0,25$ e $50 \mathrm{~mL}$ por amostra). Para HD, foram testados três tempos de destilação (100, 120 e 140 min.) e três volumes de água (1,0; 1,5 e 2,0 L por balão de 3 litros). Os óleos essenciais foram analisados por CG/EM-FID. Maiores teores de óleo essencial foram obtidos nas condições de $700 \mathrm{~W}$ por $40 \mathrm{~min}$. (3.28\%), independente do volume de água para MI, e $120 \mathrm{~min}$. com $1,0 \mathrm{~L}$ de 
água por balão para HD (3,34\%). Os compostos mais abundantes para MI (700W, por 40 min., sem água) foram o shyobunol $(26,53 \%)$ e biciclogermacreno $(4,96 \%)$ e para HD (120 min. com 1,0 L de água /balão) foram shyobunol $(24,00 \%)$ e germacreno D -4 -ol $(10,23 \%)$. Metil farnesoato $(2 E, 6 E)$ e farnesil acetato $(2 Z, 6 E)$ não foram detectados no óleo essencial extraído por HD, porém, foram detectados nas amostras extraídas por MI. Com o aumento do tempo de destilação e/ou do volume de água em HD, houve redução no conteúdo dos constituintes químicos $\beta$-elemeno (de 1,23 para $0,97 \%$ ), E-cariofileno (de 5,49 para 4,35\%), $\alpha$-humuleno (1,80 para 1,43\%), aloaromadendreno (de 1,78 para $1,44 \%$ ), biciclogermacreno (de 5,63 para 4,55\%) e germacreno D-4-ol (de 11,40 para 9,86\%). A potência, o tempo de extração e suas interações influenciaram no teor de óleo essencial obtido na extração por micro-ondas (MI). Dentro de cada potência, o maior teor de óleo essencial foi obtido no tempo mais longo de extração (40 min.), exceto para 600 W, que não apresentou diferença significativa entre 30 e $40 \mathrm{~min}$. Nas condições ótimas de extração, os teores de óleo essencial obtidos foram estatisticamente semelhantes pelo teste $\mathrm{t}$ para amostras dependentes. No entanto, a extração por micro-ondas apresenta algumas vantagens em relação a HD, como menor tempo de destilação e menor consumo de energia e água.

PALAVRAS-CHAVE: Varronia curassavica. Óleos voláteis. Destilação por micro-ondas.

\section{REFERENCES}

ADAMS, R. P. Identification of essential oil components by gas chromatography/mass spectroscopy. 4th ed., Allured: Carol Stream, 2007. 804p.

CARDOSO-UGARTE, G. A.; JUÁREZ-BECERRA, G. P.; SOSA-MORALES, M. E.; LÓPEZ-MALO, A. Microwave-assisted extraction of essential oils from herbs. Journal of Microwave Power and Electromagnetic Energy, Mechanicsville, v. 47, n. 1, p. 63-72, mar. 2013. http://dx.doi.org/10.1080/08327823.2013.11689846

CHEN, F.; DUA, X.; ZU, Y.; YANG, L.; WANG, F. Microwave-assisted method for distillation and dual extraction in obtaining essential oil, proanthocyanidins and polysaccharides by one-pot process from Cinnamomi Cortex. Separation and Purification Technology, Delft, v. 164, p. 1-11, mar. 2016. https://doi.org/10.1016/j.seppur.2016.03.018

CHEN, X.; ZHANG, Y.; ZU, Y.; FU, Y.; WANG, W. Composition and biological activities of the essential oil from Schisandra chinensis obtained by solvent-free microwave extraction. Food Science and Technology, Zurique, v. 44, p. 2047-2052, dec. 2011. https://doi.org/10.1016/j.1wt.2011.05.013

COSTA, S. S.; GARIEPY, Y.; ROCHA, S. C. S.; RAGHAVAN, V. Microwave extraction of mint essential oil - Temperature calibration for the oven. Journal of Food Engineering, Pullman, v. 126, p. 1-6, apr. 2014. https://doi.org/10.1016/j.jfoodeng.2013.10.033

FERNANDES, E. S.; PASSOS, G. F.; MEDEIROS, R.; CUNHA, F. M.; FERREIRA, J.; CAMPOS, M. M.; PIANOWSKI, L. F.; CALIXTO, J. B. Anti-inflammatory effects of compounds alpha-humulene and (-)-transcaryophyllene isolated from the essential oil of Cordia verbenacea. European Journal of Pharmacology, Amsterdam, v. 569, n. 3, p. 228-236, aug. 2007. https://doi.org/10.1016/j.ejphar.2007.04.059

GASPARINO, E. C.; BARROS, M. A. V. C. Palinotaxonomia das espécies de Cordiaceae (Boraginales) ocorrentes no Estado de São Paulo. Brazilian Journal of Botany, São Paulo, v. 32, n. 1, p. 33-55, jan. 2009. http://dx.doi.org/10.1590/S0100-84042009000100005

GAVAHIAN, M.; FARAHNAKY, A.; FARHOOSH, R.; JAVIDNIA, K.; SHAHIDI, F. Extraction of essential oils from Mentha piperita using advanced techniques: Microwave versus ohmic assisted hydrodistillation.

Food and Bioproducts Processing, Birmingham, v. 9, p. 50-58, jan. 2015.

https://doi.org/10.1016/j.fbp.2015.01.003 
GONZÁLEZ-RIVERA, J.; DUCE, C.; FALCONIERI, D.; FERRARI, C.; GHEZZI, L.; ALESSANDRA PIRAS, A.; TINE, M. R. Coaxial microwave assisted hydrodistillation of essential oils from five different herbs (lavender, rosemary, sage, fennel seeds and clove buds): Chemical composition and thermal analysis. Innovative Food Science and Emerging Technologies, Wageningen, v.33, p. 308-318, jan. 2016. https://doi.org/10.1016/j.ifset.2015.12.011

HOYOS, J. M. A.; ALVES, E.; ROZWALKA, L. C.; SOUZA, E. A.; ZEVIANI, W. M. Antifungal activity and ultrastructural alterations in Pseudocercospora griseola treated with essential oils. Ciência e Agrotecnologia, Lavras, v. 36, n. 3, p. 270-284, may 2012. http://dx.doi.org/10.1590/S1413-70542012000300002

KARAKAYA, S.; EL, S. N.; KARAGOZLU, N.; SAHIN, S.; SUMNU, G.; BAYRAMOGLU, B. Microwaveassisted hydrodistillation of essential oil from rosemary. Journal of Food Science and Technology, Mysore, v. 51, n. 6, p. 1056-1065, june 2014. https://doi.org/10.1007/s13197-011-0610-y

KHANAVI, M.; HAJIMEHDIPOOR, H.; EMADI, F.; KHANDANI, N. K. Essential oil compositions of Thymus kotschyanus Boiss. obtained by hydrodistillation and microwave oven distillation. Journal of Essential Oil Bearing Plants, India, v. 16, n. 1, p. 117-122, may 2013.

http://dx.doi.org/10.1080/0972060X.2013.764159

LI, S.; CHEN, F.; JIA, J.; LIU, Z.; GU, H.; YANG, L.; WANG, F.; YANG, F. Ionic liquid-mediated microwave-assisted simultaneous extraction and distillation of gallic acid, ellagic acid and essential oil from the leaves of Eucalyptus camaldulensis. Separation and Purification Technology, Delft, v. 168, p. 8-18, may 2016. https://doi.org/10.1016/j.seppur.2016.05.013

LI, X. J.; WANG, W.; LUO, M.; LI, C. Y.; ZU, Y. G.; MU, P. S.; FU, Y. J. Solvent-free microwave extraction of essential oil from Dryopteris fragrans and evaluation of antioxidant activity. Food Chemistry, Norwich, v. 133, n. 2, p. 437-444, jan. 2012. https://doi.org/10.1016/j.foodchem.2012.01.056

LI, Y.; FABIANO-TIXIER, A. S.; CHEMAT, F. Essential oils: From conventional to green extraction. In: Green chemistry for sustainability. Cham: Springer International Publishing, 2014, chapter 2, p. 9-21. http://dx.doi.org/10.1007/978-3-319-08449-7

MATIAS, E. F.; SANTOS, K. K. A.; ALMEIDA, T. S.; COSTA, J. G. M.; COUTINHO, H. D. M. Atividade antibacteriana in vitro de Croton campestris A., Ocimum gratissimum L. e Cordia verbenacea DC. Revista Brasileira de Biociencias, Porto Alegre, v. 8, n. 3, p. 294-298, july, 2010. Disponível <http://www.ufrgs.br/seerbio/ojs/index.php/rbb/article/view/1428> Acesso em: 5 maio de 2015.

MECCIA, G.; ROJAS, L. B.; VELASCO, J.; DÍAZ, T.; USUBILLAGA, A.; ARZOLA, J. C.; RAMOS, S. Chemical composition and antibacterial activity of the essential oil of Cordia verbenacea from the Venezuelan Andes. Natural Product Communications, Westerville, v. 4, n. 8, p. 1119-1122, aug. 2009.

MEDEIROS, R.; PASSOS, G. F.; VITOR, C. E.; MAZZUCO, T. L.; PIANOWSKI, L. F.; CAMPOS, M. M.; CALIXTO, J. B. Effect of two active compounds obtained from the essential oil of Cordia verbenacea on the acute inflammatory responses elicited by LPS in the rat paw. British Journal of Pharmacology, London, v. 151, n. 5, p. 618-627, july 2007. http://dx.doi.org/10.1038/sj.bjp.0707270

MICHIELIN, E. M. Z.; WIESE, L. P. L.; FERREIRA, E. A.; PEDROSA, R. C.; FERREIRA, S. R. S. Radicalscavenging activity of extracts from Cordia verbenacea DC obtained by different methods. Journal of Supercritical Fluids, Netherlands, v. 56, n. 1, p. 89-96, feb. 2011. https://doi.org/10.1016/j.supflu.2010.11.006

OLIVEIRA, D. M.; LUCHINI, A. C.; SEITO, L. N.; GOMES, J. C.; CRESPO-LÓPEZ, M. E.; DI STASI, L. C. Cordia verbenacea and secretion of mast cells in different animal species. Journal of Ethnopharmacology, Ireland, v. 135, n. 2, p. 463-468, may 2011. https://doi.org/10.1016/j.jep.2011.03.046 
PARISOTTO, E. B.; MICHIELIN, E. M. Z.; BISCARO, F.; FERREIRA, S. R. S.; FILHO, D. W.; PEDROSA, R. C. The antitumor activity of extracts from Cordia verbenacea D.C. obtained by supercritical fluid extraction. Journal of Supercritical Fluids, Netherlands, v. 61, p. 101-107, jan. 2012.

https://doi.org/10.1016/j.supflu.2011.08.016

PASSOS, G. F.; FERNANDES, E. S.; CUNHA, F. M.; FERREIRA, J.; PIANOWSKI, L. F.; CAMPOS, M. M.; CALIXTO, J. B. Anti-inflammatory and anti-allergic properties of the essential oil and active compounds from Cordia verbenacea. Journal of Ethnopharmacology, Ireland, v. 110, n. 2, p. 323-333, mar. 2007. https://doi.org/10.1016/j.jep.2006.09.032

PIMENTEL, S. P.; BARRELLA, G. E.; CASARIN, R. C. V.; CIRANO, F. R.; CASATI, M. Z.; FOGLIO, M. A.; FIGUEIRA, G. F.; RIBEIRO, F. V. R. Protective effect of topical Cordia verbenacea in a rat periodontitis model: immune-inflammatory, antibacterial and morphometric assays. BMC Complementary and Alternative Medicine, London, v. 12, n. 224, p. 1-8, nov. 2012. https://doi.org/10.1186/1472-6882-12-224

PINHO, L.; SOUZA, P. N. S.; SOBRINHO, E. M.; ALMEIDA, A. C.; MARTINS, E. R. Antimicrobial activity of hydroalcoholic extracts from rosemary, pepper tree, barbatimão and erva-baleeira leaves and from pequi peel meal. Ciencia Rural, Santa Maria, v. 42, n. 2, p. 326-331, feb. 2012. http://dx.doi.org/10.1590/S010384782012005000003

QI, X. L.; LI, T. T.; WEI, Z. F.; GUO, N.; LUO, M.; WANG, W.; ZUA, Y. G.; FU, Y. J.; PENG, X. Solventfree microwave extraction of essential oil from pigeon pea leaves [Cajanus cajan (L.) Mill sp.] and evaluation of its antimicrobial activity. Industrial Crops and Products, Amsterdam, v. 58, p. 322-328, july 2014. https://doi.org/10.1016/j.indcrop.2014.04.038

RANITHA, M., NOUR, A. H.; SULAIMAN, Z. A.; NOUR A. H.; THANA RAJ S. A comparative study of lemongrass (Cymbopogon Citratus) essential oil extracted by microwave-assisted hydrodistillation (MAHD) and conventional hydrodistillation (HD) method. International Journal of Chemical Engineering and Applications, Singapore, vol. 5, n. 2, p. 104-108, apr. 2014. https://doi.org/10.7763/IJCEA.2014.V5.360

RIELA, S.; BRUNO, M.; ROSSELLI, S.; SALADINO, M.L.; CAPONETTI, E.; FORMISANO, C.; SENATORE, F. A study on the essential oil of Ferulago campestris: How much does extraction method influence the oil composition? Journal of Separation Science, Weinheim, v. 34, n. 4, p. 483-492, feb. 2011. https://doi.org/10.1002/jssc.201000411

RODRIGUES, F. F. G; OLIVEIRA, L. G. S.; RODRIGUES, F. F. G.; SARAIVA, M. E.; ALMEIDA, S. C. X.; CABRAL, M. E. S.; CAMPOS, A. R.; COSTA, J. G. M. Chemical composition, antibacterial and antifungal activities of essential oil from Cordia verbenacea DC leaves. Pharmacognosy Research, Mumbai, v. 4, n. 3 , p. 161-165, july 2012. https://doi.org/10.4103/0974-8490.99080

ROGÉRIO, A. P.; ANDRADE, E. L.; LEITE, D. F. P.; FIGUEIREDO, C.; CALIXTO, J. B. Themed section: mediators and receptors in the resolution of inflammation. Preventive and therapeutic anti-inflammatory properties of the sesquiterpene $\alpha$-humulene in experimental airways allergic inflammation. British Journal of Pharmacology, London, v. 158, n. 4, p. 1074-1087, oct. 2009. https://doi.org/10.1111/j.14765381.2009.00177.x

SHAH, M.; GARG, S. K. Application of $2^{k}$ full factorial design in optimization of solvent-free microwave extraction of ginger essential oil. Journal of Engineering, Nasr City, v. 2014, p. 1-5, nov. 2014. http://dx.doi.org/10.1155/2014/828606

SILVA, A. C.; SOUZA, P. E.; MACHADO, J. C.; SILVA, B. M.; PINTO, J. E. B. P. Effectiveness of essential oils in the treatment of Colletotrichum truncatum-infected soybean seeds. Tropical Plant Pathology, Brasília, v. 37, n. 5, p. 305-313, sept. 2012. http://dx.doi.org/10.1590/S1982-56762012000500001 
SILVA, A. C.; SOUZA, P. E.; RESENDE, M. L. V.; SILVA JR., M. B.; RIBEIRO JR., P. M.; ZEVIANI, W. M. Local and systemic control of powdery mildew in eucalyptus using essential oils and decoctions from traditional Brazilian medicinal plants. Forest Pathology, Freising, v. 44, n. 2, p. 145-153, apr. 2014. http://dx.doi.org/10.1111/efp.12079

SOZMEN, F.; UYSAL, B.; OKSAL, B. S.; KOSE, E. O.; DENIZ, I. G. Chemical composition and antibacterial activity of Origanum saccatum P.H. Davis essential oil obtained by solvent-free microwave extraction: comparison with hydrodistillation. Journal of AOAC International, Gaithersburg, v. 94, n. 1, p. 243-250, jan. 2011. http://dx.doi.org/10.1111/j.1745-4565.2009.00181.x

TONGNUANCHAN, P.; BENJAKUL, S. Essential oils: extraction, bioactivities, and their uses for food preservation. Journal of Food Science, Chicago, v. 79, n. 7, p. 1231-1249, july 2014.

http://dx.doi.org/10.1111/1750-3841.12492

VAN DEN DOOL, H.; KRATZ, P. D. A generalization of the retention index system including linear temperature programmed gas-liquid partition chromatography. Journal of Chromatography A, Netherlands, v. 11, p. 463-471, aug. 1963. https://doi.org/10.1016/S0021-9673(01)80947-X

VERMA, R. S.; RAHMAN, L.; VERMA, R. K.; CHAUHAN, A.; YADAV, A. K.; SINGH, A. Essential oil composition of menthol mint (Mentha arvensis) and pepper mint (Mentha piperita) cultivars at different stages of plant growth from Kumaon region of western Himalaya. Journal of Medicinal and Aromatic Plants, Anand, v.1, n. 1, p. 13-18, jan. 2010. Disponível <https://pt.scribd.com/document/265127902/Essential-OilComposition-of-Menthol-Mint-Mentha-arvensis-and-Peppermint-Mentha-piperita-Cultivars-at-DifferentStages-of-Pla> Acesso em: 14 de abril de 2015. 